\title{
CONGRUENCE OF MINIMAL SURFACES
}

\author{
OGNIAN KASSABOV
}

\begin{abstract}
An interesting problem in classical differential geometry is to find methods to prove that two surfaces defined by different charts actually coincide up to position in space. In a previous paper we proposed a method in this direction for minimal surfaces. Here we explain not only how this method works but also how we can find the correspondence between the minimal surfaces, if they are congruent. We show that two families of minimal surfaces which are proved to be conjugate actually coincide and coincide with their associated surfaces. We also consider another family of minimal polynomial surfaces of degree 6 and we apply the method to show that some of them are congruent.
\end{abstract}

\section{INTRODUCTION}

Minimal surfaces have a long and interesting history. The name minimal corresponds to the property that each point of such a surface has a neighborhood that minimizes the area among all surfaces with the same boundary. It turns out that this is equivalent to the vanishing of the mean curvature of the surface. The first nontrivial minimal surfaces discovered were the catenoid and the helicoid. After that, for many years no new minimal surfaces were found. From the investigations of Weierstrass and Enneper in the 19th century we know that any minimal surface can be found at least locally from a complex minimal curve, see e.g. [2].

Nowadays the minimal surfaces are important in many areas such as architecture, material science, aviation, biology. Because of their minimizing property, these surfaces can be also interesting in CAD research. Note that, for the moment, in this area it is more important to consider polynomial minimal surfaces. The problem is that such surfaces are probably not very numerous in small degrees. In [1] it is shown that up to homothety and position in the space, the classical Enneper surface is the unique polynomial minimal surface of degree 3 .

In [6] and [7] some families of polynomial minimal surfaces of degrees 6 and 5 are introduced. In [5] we showed a relation among these families. To do this, we cannot use the classical method used in [1] for degree 3. We need a new method developed thanks to a new approach to minimal surfaces, proposed by Ganchev [3].

Here we explain with examples how this method works. Namely, in section 4 we prove that the above mentioned families of polynomial surfaces of degree 6 from [6] contain many congruent surfaces and that all the surfaces in these families coincide up to homothety and position in space with the classical Enneper surface. We also show how one can look for an explicit relation between congruent minimal surfaces. Note that the construction of these

Key words and phrases. Minimal surface, canonical principal parameters, associated surfaces.

2010 Mathematics Subject Classification: 53A10.

This research is supported by the National Science Fund of the Bulgarian Ministry of Education and Science under Grant DFNI-T01/0001, 2012. 
examples in [6] is based on a theorem for the coefficients of a minimal polynomial surface of degree six, but two of the equations in this theorem as formulated in [6] are not exact. In section 3, we give the correct version of the theorem. Actually, the construction of the above mentioned examples is not affected by the wrong equations. Unfortunately in [6] another general family is obtained by using one of the wrong equations and the surfaces in it are not minimal. So in section 5 we construct a new large family of polynomial minimal surfaces of degree 6 and we prove some relations between its surfaces.

\section{Preliminaries}

Let $S$ be a regular surface defined by the parametric equation

$$
\boldsymbol{x}=\boldsymbol{x}(u, v)=\left(x_{1}(u, v), x_{2}(u, v), x_{3}(u, v)\right), \quad(u, v) \in U \subset \mathbb{R}^{2} .
$$

Denote the derivatives of the vector function $\boldsymbol{x}=\boldsymbol{x}(u, v)$ by $\boldsymbol{x}_{u}, \boldsymbol{x}_{v}, \boldsymbol{x}_{u u}, \boldsymbol{x}_{u v}, \boldsymbol{x}_{v v}$. The unit normal to the surface is defined by

$$
\boldsymbol{U}=\frac{\boldsymbol{x}_{u} \times \boldsymbol{x}_{v}}{\left|\boldsymbol{x}_{u} \times \boldsymbol{x}_{v}\right|}
$$

Then the coefficients of the first and the second fundamental form are given resp. by

$$
E=\boldsymbol{x}_{u}^{2}, \quad F=\boldsymbol{x}_{u} \boldsymbol{x}_{v}, \quad G=\boldsymbol{x}_{v}^{2},
$$

and

$$
L=\boldsymbol{U} x_{u u}, \quad M=\boldsymbol{U} x_{u v}, \quad N=\boldsymbol{U} x_{v v} .
$$

Recall that the parameters of the surface are called isothermal, if $E=G, F=0$.

The Gauss curvature $K$ and the mean curvature $H$ of a surface $S$ are defined by

$$
K=\frac{L N-M^{2}}{E G-F^{2}}, \quad H=\frac{E N-2 F M+G L}{2\left(E G-F^{2}\right)} .
$$

The surface $S$ is called minimal if its mean curvature vanishes identically. In this case the Gauss curvature is negative. The normal curvature $\nu$ of a minimal surface $S$ is defined to be the function $\nu=\sqrt{-K}$, see [3]. This is exactly the positive principal curvature of $S$.

Let us now consider a minimal surface defined in isothermal parameters. Then it can be considered as a real part of a complex curve. More precisely, let $f(z)$ and $g(z)$ be two holomorphic functions. Define the Weierstrass complex curve $\Psi(z)$ by

$$
\boldsymbol{\Psi}(z)=\int_{z_{0}}^{z}\left(\frac{1}{2} f(z)\left(1-g^{2}(z)\right), \frac{i}{2} f(z)\left(1+g^{2}(z)\right), f(z) g(z)\right) d z .
$$

Then $\boldsymbol{\Psi}(z)$ is a minimal curve, i.e. $\boldsymbol{\Psi}^{\prime 2}(z)=0$, and its real and imaginary parts $\boldsymbol{x}(u, v)$ and $\boldsymbol{y}(u, v)$ define two minimal surfaces in isothermal parametrizations. We say that these two minimal surfaces are conjugate. Moreover, every minimal surface can be obtained at least locally as the real part of a Weierstrass minimal curve.

Example. Taking $f(z)=e^{z}, g(z)=e^{-z}$ we obtain a Weierstrass minimal curve whose real part is the catenoid

$$
\boldsymbol{x}(u, v)=(\cosh u \cos v,-\cosh u \sin v, u)
$$

and the imaginary part is the helicoid

$$
\boldsymbol{y}(u, v)=(\sinh u \sin v, \sinh u \cos v, v) .
$$


Given two conjugate minimal surfaces $\boldsymbol{x}(u, v)$ and $\boldsymbol{y}(u, v)$ we can define the associated family

$$
S_{t} \quad: \quad \boldsymbol{a s s o c}_{t}(u, v)=\boldsymbol{x}(u, v) \cos t+\boldsymbol{y}(u, v) \sin t .
$$

Then for any real number $t$ the surface $S_{t}$ is also minimal and has the same first fundamental form as $S$.

It is easy to verify that the coefficients of the first fundamental form of the associated family defined in the above way via the Weierstrass formula with functions $f(z), g(z)$ are given by

$$
E=\frac{1}{4}|f|^{2}\left(1+|g|^{2}\right)^{2} \quad F=0 \quad G=\frac{1}{4}|f|^{2}\left(1+|g|^{2}\right)^{2}
$$

and the normal curvature is

$$
\nu=\frac{4\left|g^{\prime}\right|}{|f|\left(1+|g|^{2}\right)^{2}}
$$

see [4, Theorem 22.33].

A new approach to minimal surfaces was proposed by Ganchev in [3]. He introduced special principal parameters and called them canonical principal parameters. The surface being parametrized with them, the coefficients of its fundamental forms are given by

$$
\begin{array}{ccr}
E=\frac{1}{\nu}, & F=0, & G=\frac{1}{\nu} \\
L=1, & M=0, & N=-1 .
\end{array}
$$

Moreover, the surface in canonical principal parametrization is the real part of a Weierstrass minimal curve generated by some functions $f(z), g(z)$ with $f(z)=-1 / g^{\prime}(z)$, i.e. it is the real part of the special Weierstrass curve

$$
\boldsymbol{\Phi}(z)=-\int_{z_{0}}^{z}\left(\frac{1-g^{2}(z)}{2 g^{\prime}(z)}, \frac{i\left(1+g^{2}(z)\right)}{2 g^{\prime}(z)}, \frac{g(z)}{g^{\prime}(z)}\right) d z
$$

The following theorem is of great importance:

Theorem 2.1. 3] If a surface is parametrized with canonical principal parameters, then its normal curvature satisfies the differential equation

$$
\Delta \ln \nu+2 \nu=0 .
$$

Conversely, for any solution $\nu(u, v)$ of this equation (with $\nu_{u} \nu_{v} \neq 0$ ), there exists a unique (up to position in space) minimal surface with normal curvature $\nu(u, v),(u, v)$ being canonical principal parameters. Moreover, the canonical principal parameters $(u, v)$ are determined uniquely up to the following transformations

$$
\begin{aligned}
& u=\varepsilon \bar{u}+a, \\
& v=\varepsilon \bar{v}+b, \quad \varepsilon= \pm 1, a=\text { const., } b=\text { const. }
\end{aligned}
$$

We will also use the following results:

Theorem 2.2. [5] Let the minimal surface $S$ be defined by the real part of the Weierstrass minimal curve (2.1). Any solution of the differential equation

$$
\left(z^{\prime}(w)\right)^{2}=-\frac{1}{f(z(w)) g^{\prime}(z(w))} .
$$


defines a transformation of the isothermal parameters of $S$ to canonical principal parameters. Moreover the function $\tilde{g}(w)$ that defines $S$ via the Ganchev formula is given by $\tilde{g}(w)=g(z(w))$.

Theorem 2.3. [5] Let the holomorphic function $g(z)$ generate a minimal surface in canonical principal parameters, i.e. via the Ganchev formula. Then, for an arbitrary complex number $\alpha$, and for an arbitrary real number $\varphi$, any of the functions

$$
e^{i \varphi} \frac{\alpha+g(z)}{1-\bar{\alpha} g(z)}
$$

generates the same surface in canonical principal parameters (up to position in space). Conversely, any function that generates this surface (up to position in space) in canonical principal parameters has the above form.

More precisely, from the proof of the last theorem we see that, up to a translation, the surface generated by the function (2.6) is obtained from the surface generated by $g(z)$ after a rotation with the matrix $A B$, where

$$
A=\left(\begin{array}{ccc}
\cos \varphi & -\sin \varphi & 0 \\
\sin \varphi & \cos \varphi & 0 \\
0 & 0 & 1
\end{array}\right), \quad B=\left(\begin{array}{ccc}
\frac{1-a^{2}+b^{2}}{1+a^{2}+b^{2}} & -\frac{2 a b}{1+a^{2}+b^{2}} & -\frac{2 a}{1+a^{2}+b^{2}} \\
-\frac{2 a b}{1+a^{2}+b^{2}} & \frac{1+a^{2}-b^{2}}{1+a^{2}+b^{2}} & -\frac{2 b}{1+a^{2}+b^{2}} \\
\frac{2 a}{1+a^{2}+b^{2}} & \frac{2 b}{1+a^{2}+b^{2}} & \frac{1-a^{2}-b^{2}}{1+a^{2}+b^{2}}
\end{array}\right)
$$

and $\alpha=a+i b$. In particular, if $\alpha=0$ this is a rotation by the angle $\varphi$ about the third axis.

Theorem 2.4. [5] For arbitrary nonzero complex numbers a, b, the minimal surfaces generated via the Weierstrass formula by the pairs of functions

$$
f(z)=a z^{k}, \quad g(z)=b z^{n}, \quad a, b \in \mathbb{C} \backslash\{0\}, \quad k, n \in \mathbb{N} .
$$

coincide up to position in space and homothety.

\section{Parametric minimal surfaces of Degree SiX.}

It is shown in [6] that if a parametric minimal surface of degree 6 is presented in isothermal parameters, then it has the form

$$
\begin{aligned}
\boldsymbol{r}(u, v)= & \boldsymbol{a}\left(u^{6}-15 u^{4} v^{2}+15 u^{2} v^{4}-v^{6}\right)+\boldsymbol{b}\left(3 u^{5} v-10 u^{3} v^{3}+3 u v^{5}\right) \\
& +\boldsymbol{c}\left(u^{5}-10 u^{3} v^{2}+5 u v^{4}\right)+\boldsymbol{d}\left(v^{5}-10 u^{2} v^{3}+5 u^{4} v\right) \\
& +\boldsymbol{e}\left(u^{4}-6 u^{2} v^{2}+v^{4}\right)+\boldsymbol{f} u v\left(u^{2}-v^{2}\right)+\boldsymbol{g} u\left(u^{2}-3 v^{2}\right)+\boldsymbol{h} v\left(v^{2}-3 u^{2}\right) \\
& +\boldsymbol{i}\left(u^{2}-v^{2}\right)+\boldsymbol{j} u v+\boldsymbol{k} u+\boldsymbol{l} v+\boldsymbol{m}
\end{aligned}
$$

where $\boldsymbol{a}, \boldsymbol{b}, \boldsymbol{c}, \boldsymbol{d}, \boldsymbol{e}, \boldsymbol{f}, \boldsymbol{g}, \boldsymbol{h}, \boldsymbol{i}, \boldsymbol{j}, \boldsymbol{k}, \boldsymbol{l}, \boldsymbol{m}$ are coefficient vectors. Then the authors of [6] formulate and give a sketch of the proof of a theorem about the relation between these coefficients so that the surface be minimal. Actually, two of the equations in this theorem are not exact. Namely, the statement must look as follows 
Theorem 3.1. The harmonic polynomial surface (3.1) is a minimal surface if and only if its coefficient vectors satisfy the following system of equations

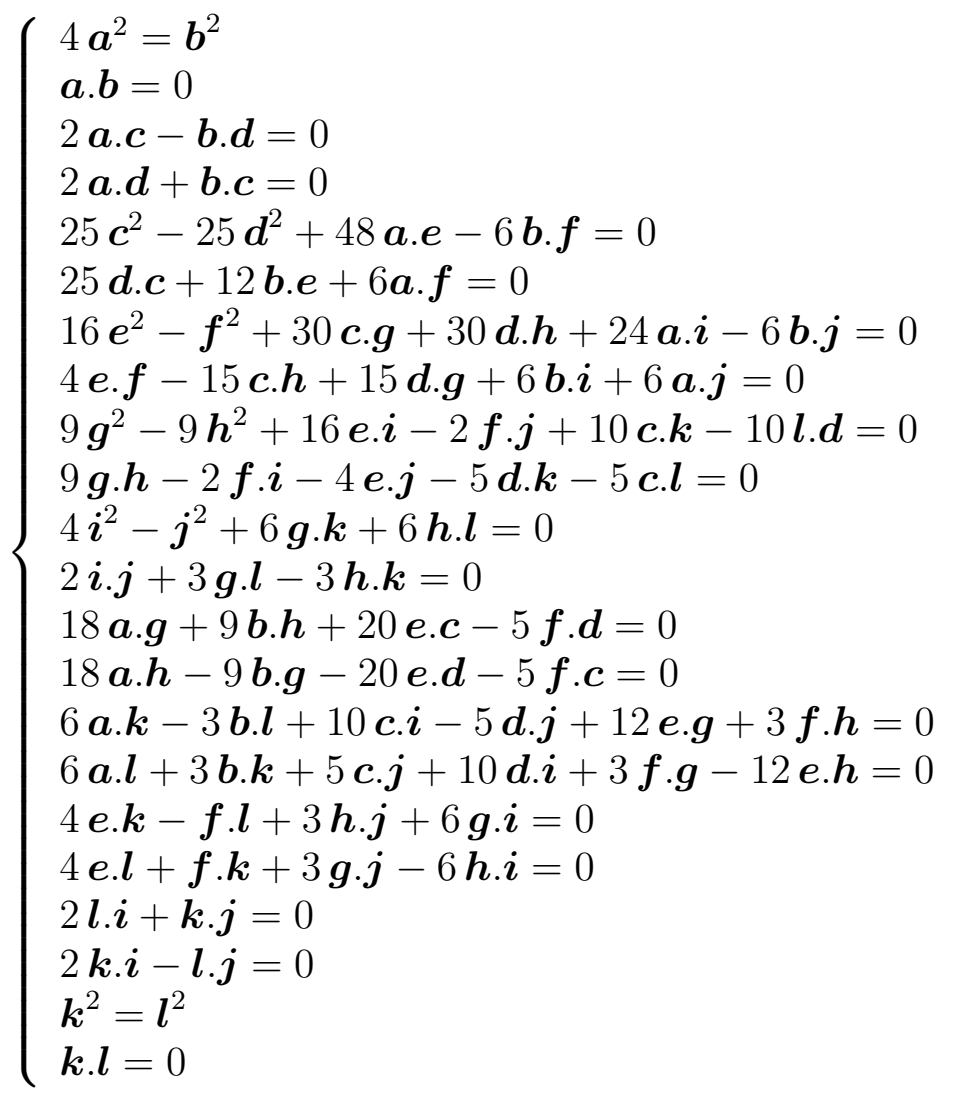

The proof is based on the vanishing of the coefficients of $u^{\alpha} v^{\beta}$ in the power expansion of the isothermal condition $E=G, F=0$. The differences with the theorem as formulated in [6] are in the seventh and the twelfth equations.

Using the first four equations of the system (3.2), we can suppose that the coefficient vectors are $\boldsymbol{a}=\left(a_{1}, a_{2}, 0\right), \boldsymbol{b}=\left(-2 a_{2}, 2 a_{1}, 0\right), \boldsymbol{c}=\left(c_{1}, c_{2}, c_{3}\right), \boldsymbol{d}=\left(-c_{2}, c_{1}, d_{3}\right), \boldsymbol{e}=$ $\left(e_{1}, e_{2}, e_{3}\right), \boldsymbol{f}=\left(f_{1}, f_{2}, f_{3}\right), \boldsymbol{g}=\left(g_{1}, g_{2}, g_{3}\right), \boldsymbol{h}=\left(h_{1}, h_{2}, h_{3}\right), \boldsymbol{i}=\left(i_{1}, i_{2}, i_{3}\right), \boldsymbol{j}=\left(j_{1}, j_{2}, j_{3}\right)$, $\boldsymbol{k}=\left(k_{1}, k_{2}, k_{3}\right), \boldsymbol{l}=\left(l_{1}, l_{2}, l_{3}\right), \boldsymbol{m}=(0,0,0)$, see [6].

\section{First example.}

If we suppose $\boldsymbol{c}=\boldsymbol{d}=\boldsymbol{g}=\boldsymbol{h}=\boldsymbol{k}=\boldsymbol{l}=\boldsymbol{o}, j_{1}=2 i_{2}, j_{2}=-2 i_{1}$, we obtain a family of minimal surfaces, depending on four shape parameters $a_{1}, a_{2}, i_{1}, i_{2}$. Two special cases are studied in [6]. Namely, if $a_{2}=i_{2}=0$ the surface is

$$
\boldsymbol{r}_{1}\left[a_{1}, i_{1}\right](u, v)=\left(X_{1}(u, v), Y_{1}(u, v), Z_{1}(u, v)\right)
$$

where

$$
\begin{aligned}
& X_{1}(u, v)=a_{1}\left(u^{6}-15 u^{4} v^{2}+15 u^{2} v^{4}-v^{6}\right)+i_{1}\left(u^{2}-v^{2}\right) \\
& Y_{1}(u, v)=2 a_{1}\left(3 u^{5} v-10 u^{3} v^{3}+3 u v^{5}\right)-2 i_{1} u v \\
& Z_{1}(u, v)=\sqrt{\frac{3}{2}} \sqrt{\left|a_{1} i_{1}\right|-a_{1} i_{1}}\left(u^{4}-6 u^{2} v^{2}+v^{4}\right)-2 \sqrt{6} \sqrt{\left|a_{1} i_{1}\right|+a_{1} i_{1}} u v\left(u^{2}-v^{2}\right)
\end{aligned}
$$

and if $a_{1}=i_{1}=0$, the surface is

$$
\boldsymbol{r}_{2}\left[a_{2}, i_{2}\right](u, v)=\left(X_{2}(u, v), Y_{2}(u, v), Z_{2}(u, v)\right)
$$


where

$$
\begin{aligned}
& X_{2}(u, v)=-2 a_{2}\left(3 u^{5} v-10 u^{3} v^{3}+3 u v^{5}\right)+2 i_{2} u v \\
& Y_{2}(u, v)=a_{2}\left(u^{6}-15 u^{4} v^{2}+15 u^{2} v^{4}-v^{6}\right)+i_{2}\left(u^{2}-v^{2}\right) \\
& Z_{2}(u, v)=\sqrt{\frac{3}{2}} \sqrt{\left|a_{2} i_{2}\right|-a_{2} i_{2}}\left(u^{4}-6 u^{2} v^{2}+v^{4}\right)-2 \sqrt{6} \sqrt{\left|a_{2} i_{2}\right|+a_{2} i_{2}} u v\left(u^{2}-v^{2}\right)
\end{aligned}
$$

The following theorem is proved in [6]:

Theorem 4.1. The surfaces defined by the charts $\boldsymbol{r}_{1}\left[a_{1}, i_{1}\right](u, v)$ and $\boldsymbol{r}_{2}\left[-a_{1}, i_{1}\right](u, v)$ are conjugate minimal surfaces.

The figures bellow represent the surfaces $\boldsymbol{r}_{1}[1,500](u, v)$ and $\boldsymbol{r}_{2}[-1,500](u, v)$ for $u, v \in$ $[-4,4]$.

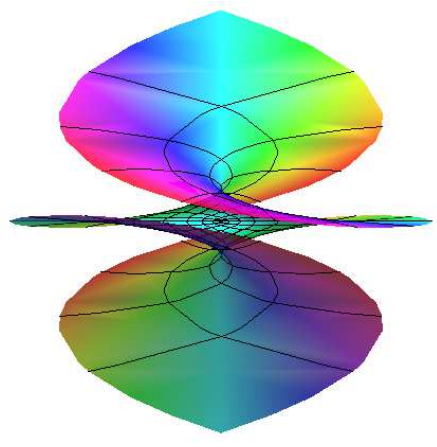

Fig. 4.1: $\boldsymbol{r}_{1}[1,500](u, v)$

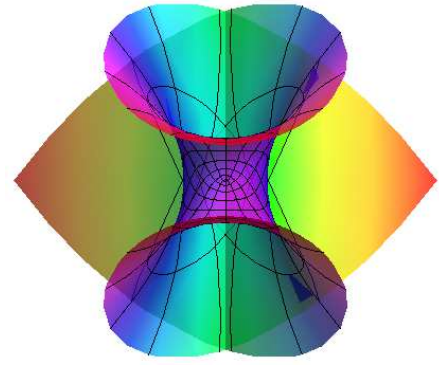

Fig. 4.2: $\boldsymbol{r}_{1}[1,-500](u, v)$

It turns out that the conjugate surfaces in Theorem 4.1 actually coincide. We will see that moreover they coincide with any surface of their associated family. Namely, we have

Theorem 4.2. For any real number $t$ the surface

$$
S_{t} \quad: \quad \boldsymbol{a s s o c}_{t}\left[a_{1}, i_{1}\right](u, v)=\boldsymbol{r}_{1}\left[a_{1}, i_{1}\right] \cos t+\boldsymbol{r}_{2}\left[-a_{1}, i_{1}\right](u, v) \sin t
$$

coincides up to position in space with that defined by $\boldsymbol{r}_{1}\left[a_{1}, i_{1}\right](u, v)=\boldsymbol{a s s o c}_{0}\left[a_{1}, i_{1}\right](u, v)$.

Proof. Suppose for example that $a_{1} i_{1}>0$. We will change the parameters of $S_{t}$ to canonical principal ones. First we need the functions that generate $\boldsymbol{a s s}_{\boldsymbol{o}} \boldsymbol{c}_{t}\left[a_{1}, i_{1}\right](u, v)$ via the Weierstrass formula. They can be found from the well known relations

$$
f_{t}=\varphi_{1}-i \varphi_{2}, \quad g_{t}=\frac{\varphi_{3}}{\varphi_{1}-i \varphi_{2}}
$$

where

$$
\left(\varphi_{1}, \varphi_{2}, \varphi_{3}\right)=\left(\boldsymbol{a s s o c}_{t}\right)_{u}-i\left(\boldsymbol{a s s o c}_{t}\right)_{v}
$$

see e.g. 4], Lemma 22.31. In our case these equalities give

$$
f_{t}(z)=4 i_{1} e^{-i t} z, \quad g_{t}(z)=\frac{\sqrt{3} a_{1} i}{\sqrt{a_{1} i_{1}}} z^{2} .
$$

With these functions a solution of (2.5) is

$$
z(w)=\frac{\sqrt{(1+i) e^{i t / 2} w}}{\sqrt{2}\left(3 a_{1} i_{1}\right)^{1 / 8}} .
$$


So we change $z$ in $g_{t}(z)$ by $z(w)$ and we obtain that the function

$$
\tilde{g}_{t}(w)=\frac{(-1+i) 3^{1 / 4} a_{1} e^{i t / 2} w}{2\left(a_{1} i_{1}\right)^{3 / 4}}
$$

generates $S_{t}$ in canonical principal parameters. Then, using (2.3) we obtain the normal curvature in canonical principal parameters to be (recall that $t$ is real)

$$
\tilde{\nu}=\frac{4\left|\tilde{g}_{t}^{\prime}\right|^{2}}{\left(1+\left|\tilde{g}_{t}\right|^{2}\right)^{2}}=8 \sqrt{3} \frac{\left|\frac{a_{1}}{\left(a_{1} i_{1}\right)^{3 / 4}}\right|^{2}}{\left(2+\sqrt{3}\left|\frac{a_{1}}{\left(a_{1} i_{1}\right)^{3 / 4}} w\right|^{2}\right)^{2}}
$$

Since this number does not depend on $t$, Theorem 2.1 implies that any surface $S_{t}$ is congruent with every other surface in the associated family and in particular to $S_{0}$.

In the same way we can prove

Theorem 4.3. The minimal surfaces defined by the charts $\boldsymbol{r}_{1}\left[a_{1}, i_{1}\right](u, v), \boldsymbol{r}_{1}\left[a_{1},-i_{1}\right](u, v)$, $\boldsymbol{r}_{1}\left[-a_{1}, i_{1}\right](u, v), \boldsymbol{r}_{1}\left[-a_{1},-i_{1}\right](u, v)$ are congruent.

Corollary 4.4. All the minimal surfaces from the associated families assoc $_{t}\left[a_{1}, i_{1}\right](u, v)$, $\boldsymbol{a s s o c}_{t}\left[a_{1},-i_{1}\right](u, v)$, $\boldsymbol{a s s o c}_{t}\left[-a_{1}, i_{1}\right](u, v)$, $\boldsymbol{a s s o c}_{t}\left[-a_{1},-i_{1}\right](u, v)$ are congruent.

Of course, the proof of Theorem 4.2 given above hides the relation between the charts $\boldsymbol{r}_{1}\left[a_{1}, i_{1}\right](u, v)$ and $\boldsymbol{a s s o c}_{t}\left[a_{1}, i_{1}\right](u, v)$. To show this relation we first remark that we can find an alternative end of the proof of Theorem 4.2 if we notice that the generating functions $\tilde{g}_{0}(z)$ and $\tilde{g}_{t}(z)$ satisfy

$$
\tilde{g}_{t}(z)=e^{\frac{i t}{2}} g_{0}(z) .
$$

Indeed, the last equality and Theorem 2.3 imply the conclusion of Theorem 4.2 . Moreover in view of (4.4) and Theorem 2.3 we can say that up to a translation $S_{t}$ is obtained from $S_{0}$ with a rotation of angle $t / 2$ around the third axis. To realize this rotation we first obtain the Weierstrass minimal curves of $\boldsymbol{r}_{1}\left[a_{1}, i_{1}\right](u, v)$ and $\boldsymbol{a s s o c}_{t}\left[a_{1}, i_{1}\right](u, v)$. By (2.1), (4.3), and the definitions of $\boldsymbol{r}_{1}\left[a_{1}, i_{1}\right](u, v)$ and $\boldsymbol{r}_{2}\left[-a_{1}, i_{1}\right](u, v)$, these curves are

$$
\begin{gathered}
\left(z^{2}\left(i_{1}+a_{1} z^{4}\right), i z^{2}\left(i_{1}-a_{1} z^{4}\right), i \sqrt{3 a_{1} i_{1}} z^{4}\right) \\
\left(e^{-i t} z^{2}\left(i_{1}+a_{1} z^{4}\right), i e^{-i t} z^{2}\left(i_{1}-a_{1} z^{4}\right), i e^{-i t} \sqrt{3 a_{1} i_{1}} z^{4}\right)
\end{gathered}
$$

respectively. So we see that the transformation from the chart (4.5) to the chart (4.6) can be made without any translation, but we have to make the change of the parameters $z \rightarrow e^{-i t / 4} z$ in (4.5). So we obtain the following parametrization of the minimal curve defined by (4.5):

$$
\left(e^{-i t / 2} z^{2}\left(i_{1}+a_{1} e^{-i t} z^{4}\right), i e^{-i t / 2} z^{2}\left(i_{1}-a_{1} e^{-i t} z^{4}\right), i e^{-i t} \sqrt{3 a_{1} i_{1}} z^{4}\right)
$$

Now the rotation of the last chart with angle $t / 2$ around the third axis gives (4.6). The real form of the above change of parameters is

$$
u \rightarrow u \cos \frac{t}{4}+v \sin \frac{t}{4} \quad v \rightarrow-u \sin \frac{t}{4}+v \cos \frac{t}{4} .
$$

As a result we obtain 
Theorem 4.5. For any real number $t$ the chart assoc $_{t}\left[a_{1}, i_{1}\right](u, v)$ is obtained from $\boldsymbol{r}_{1}\left[a_{1}, i_{1}\right](u, v)$ with a change of the parameters (4.7) and then a rotation with angle $t / 2$ aroud the third axis.

Remark also that from (4.3) and Theorem 2.4 we obtain

Theorem 4.6. For any real number $t$ and for arbitrary values of the shape parameters $a_{1}, i_{1}$, the surface (4.1) coincides up to position in space and homothety with the surface generated via the Weierstrass formula by the functions

$$
f(z)=z, \quad g(z)=z^{2} .
$$

In particular, Theorem 4.6 says that all the surfaces in the families $\boldsymbol{r}_{1}\left[a_{1}, i_{1}\right](u, v)$ and $\boldsymbol{r}_{2}\left[a_{2}, i_{2}\right](u, v)$ coincide up to position in space and homothety.

At the end of this section we note that, for any values of the shape parameters, the function $\tilde{g}(z)$ that generates the considered surfaces has the form $\tilde{g}(z)=C z$ with a complex constant $C$, just like the surfaces homothetic to the Enneper surface. This looks strange for a polynomial surface of degree 6 . Now we are obliged to take a more careful look on the equations of $\boldsymbol{r}_{1}\left[a_{1}, i_{1}\right](u, v)$ and $\boldsymbol{r}_{2}\left[a_{2}, i_{2}\right](u, v)$. We remark that they are all homothetic to the Enneper surface. If for example $a_{1} i_{1}>0$, to realize the correspondence it suffices to make the change of the parameters

$$
u^{2}-v^{2} \rightarrow \sqrt{\frac{i_{1}}{a_{1}}} U \quad 2 u v \rightarrow \sqrt{\frac{i_{1}}{a_{1}}} V .
$$

\section{SECOND EXAMPLE.}

In this section we suppose that $\boldsymbol{g}=\boldsymbol{h}=\boldsymbol{i}=\boldsymbol{j}=\boldsymbol{k}=\boldsymbol{l}=\boldsymbol{o}$. Then, with the assumptions from the end of section 3 , the system (3.2) is equivalent to

$$
\left\{\begin{array}{l}
25 \boldsymbol{c}^{2}-25 \boldsymbol{d}^{2}+48 \boldsymbol{a} . \boldsymbol{e}-6 \boldsymbol{b} . \boldsymbol{f}=0 \\
25 \boldsymbol{c} . \boldsymbol{d}+12 \boldsymbol{b} . \boldsymbol{e}+6 \boldsymbol{a} . \boldsymbol{f}=0 \\
16 \boldsymbol{e}^{2}-\boldsymbol{f}^{2}=0 \\
\boldsymbol{e} \cdot \boldsymbol{f}=0 \\
4 \boldsymbol{c} . \boldsymbol{e}-\boldsymbol{d} \cdot \boldsymbol{f}=0 \\
4 \boldsymbol{d} \cdot \boldsymbol{e}+\boldsymbol{c} . \boldsymbol{f}=0
\end{array}\right.
$$

Suppose in addition that the vectors $\boldsymbol{e}$ and $\boldsymbol{f}$ lie in $\operatorname{span}\{\boldsymbol{a}, \boldsymbol{b}\}$. If the surface is not part of a plane, then, using (5.1) $3-(5.1)_{6}$, we find $\boldsymbol{e}=\left(e_{1}, e_{2}, 0\right), \boldsymbol{f}=\left(4 e_{2},-4 e_{1}, 0\right)$. So (5.1) takes the form

$$
\left\{\begin{array}{l}
25\left(c_{3}^{2}-d_{3}^{2}\right)+96\left(a_{1} e_{1}+a_{2} e_{2}\right)=0 \\
25 c_{3} d_{3}+48\left(a_{1} e_{2}-a_{2} e_{1}\right)=0 \\
c_{1} e_{1}+c_{2} e_{2}=0 \\
c_{1} e_{2}-c_{2} e_{1}=0
\end{array}\right.
$$

If $e_{1}=e_{2}=0$, then the surface is part of a plane. So we assume that $\left(e_{1}, e_{2}\right) \neq(0,0)$. Then, from (5.2) we obtain $c_{1}=c_{2}=0$,

$$
e_{1}=\frac{25\left(-a_{1} c_{3}^{2}+2 a_{2} c_{3} d_{3}+a_{1} d_{3}^{2}\right)}{96\left(a_{1}^{2}+a_{2}^{2}\right)} \quad e_{2}=\frac{25\left(-a_{2} c_{3}^{2}-2 a_{1} c_{3} d_{3}+a_{2} d_{3}^{2}\right)}{96\left(a_{1}^{2}+a_{2}^{2}\right)}
$$


So, as in section 4 , we obtain a family of minimal surfaces depending on the four shape parameters $a_{1}, a_{2}, c_{3}, d_{3}$ :

$$
\boldsymbol{s}\left[a_{1}, a_{2}, c_{3}, d_{3}\right](u, v)=(X(u, v), Y(u, v), Z(u, v))
$$

where

$$
\begin{aligned}
X(u, v)= & a_{1}\left(u^{6}-15 u^{4} v^{2}+15 u^{2} v^{4}-v^{6}\right)-2 a_{2} u v\left(3 u^{4}-10 u^{2} v^{2}+3 v^{4}\right) \\
& -\frac{25\left(a_{1} c_{3}^{2}-2 a_{2} c_{3} d_{3}-a_{1} d_{3}^{3}\right)}{96\left(a_{1}^{2}+a_{2}^{2}\right)}\left(u^{4}-6 u^{2} v^{2}+v^{4}\right)-\frac{25\left(a_{2} c_{3}^{2}+2 a_{1} c^{3} d_{3}-a_{2} d_{3}^{2}\right)}{24\left(a_{1}^{2}+a_{2}^{2}\right)} u v\left(u^{2}-v^{2}\right) \\
Y(u, v)= & 2 a_{1} u v\left(3 u^{4}-10 u^{2} v^{2}+3 v^{4}\right)+a_{2}\left(u^{6}-15 u^{4} v^{2}+15 u^{2} v^{4}-v^{6}\right) \\
& +\frac{25\left(a_{1} c_{3}^{2}-2 a_{2} c_{3} d_{3}-a_{1} d_{3}^{3}\right)}{24\left(a_{1}^{2}+a_{2}^{2}\right)} u v\left(u^{2}-v^{2}\right)-\frac{25\left(a_{1} c_{3}^{2}-2 a_{2} c_{3} d_{3}-a_{1} d_{3}^{3}\right)}{96\left(a_{1}^{2}+a_{2}^{2}\right)}\left(u^{4}-6 u^{2} v^{2}+v^{4}\right) \\
Z(u, v)= & c_{3} u\left(u^{4}-10 u^{2} v^{2}+5 v^{4}\right)+d_{3} v\left(5 u^{4}-10 u^{2} v^{2}+v^{4}\right),
\end{aligned}
$$

Now we shall consider the surfaces defined by

$$
\boldsymbol{s}_{1}\left[a_{1}, c_{3}\right]=\boldsymbol{s}\left[a_{1}, 0, c_{3}, 0\right](u, v)
$$

and

$$
\boldsymbol{s}_{2}\left[a_{2}, d_{3}\right]=\boldsymbol{s}\left[0, a_{2}, 0, d_{3}\right](u, v)
$$

Using (4.2) we obtain the functions that generate these surfaces via the Weierstrass formula. They are respectively

$$
\begin{array}{ll}
f_{1}(z)=-\frac{25 c_{3}^{2}}{12 a_{1}} z^{3} & g_{1}(z)=-\frac{12 a_{1}}{5 c_{3}} z, \\
f_{2}(z)=-\frac{25 d_{3}^{2}}{12 a_{2}} i z^{3} & g_{2}(z)=\frac{12 a_{2}}{5 d_{3}} z .
\end{array}
$$

From (2.1) and (5.3) we find the Weierstrass minimal curve, whose real part is $\boldsymbol{s}_{1}\left[a_{1}, c_{3}\right]$ :

$$
\Psi_{1}(z)=\left(\left(a_{1} z^{2}-\frac{25 c_{3}^{2}}{96 a_{1}}\right) z^{4},-i\left(a_{1} z^{2}+\frac{25 c_{3}^{2}}{96 a_{1}}\right) z^{4}, c_{3} z^{5}\right)
$$

Then it is easy to check that the imaginary part of $\Psi_{1}(z)$ is $s_{2}\left[-a_{1}, c_{3}\right]$. Hence we have

Theorem 5.1. The minimal surfaces defined by the charts $\boldsymbol{s}_{1}\left[a_{1}, c_{3}\right](u, v)$ and $\boldsymbol{s}_{2}\left[-a_{1}, c_{3}\right](u, v)$ are conjugate.

Concerning the pairs (5.3) and (5.4), we take the following solutions of the system (2.5) for the transition to canonical principal parameters:

$$
z_{1}(w)=\left(\frac{i \sqrt{5} w}{2 \sqrt{c_{3}}}\right)^{2 / 5} \quad z_{2}(w)=i\left(\frac{i \sqrt{5} w}{2 \sqrt{d_{3}}}\right)^{2 / 5}
$$

respectively. Hence the functions

$$
\tilde{g}_{1}(z)=-\frac{6.2^{3 / 5} a_{1}}{5^{4 / 5} c_{3}}\left(\frac{i w}{\sqrt{c_{3}}}\right)^{2 / 5} \quad \tilde{g}_{2}(z)=\frac{6 i .2^{3 / 5} a_{2}}{5^{4 / 5} d_{3}}\left(\frac{i w}{\sqrt{d_{3}}}\right)^{2 / 5}
$$

generate the surfaces in canonial principal parameters. Hence it is clear that the normal curvatures of $\boldsymbol{s}_{1}\left[a_{1}, c_{3}\right](u, v)$ and $\boldsymbol{s}_{2}\left[-a_{1}, c_{3}\right](u, v)$ coincide, and so we have

Theorem 5.2. The conjugate minimal surfaces defined by the charts $\boldsymbol{s}_{1}\left[a_{2}, c_{3}\right](u, v)$ and $\boldsymbol{s}_{2}\left[-a_{1}, c_{3}\right](u, v)$ are congruent. 
Corollary 5.3. Any two minimal surfaces from the associated family

$$
\boldsymbol{s}_{1}\left[a_{1}, c_{3}\right](u, v) \cos t+\boldsymbol{s}_{2}\left[-a_{1}, c_{3}\right](u, v) \sin t \quad t \in[0, \pi / 2]
$$

are congruent.

To find the exact relation between the conjugate charts $\boldsymbol{s}_{1}\left[a_{1}, c_{3}\right](u, v)$ and $\boldsymbol{s}_{2}\left[-a_{1}, c_{3}\right](u, v)$, we can use the same arguments as in section 4 . Then we can see that the chart $\boldsymbol{s}_{2}\left[-a_{1}, c_{3}\right](u, v)$ is obtained from $s_{1}\left[a_{1}, c_{3}\right](u, v)$ with the change of the parameters $u \rightarrow v, v \rightarrow-u$ and then a rotation with angle $\pi / 2$ about the third axis.

Of course, this is not the only way to obtain $\boldsymbol{s}_{2}\left[-a_{1}, c_{3}\right](u, v)$ from $\boldsymbol{s}_{1}\left[a_{1}, c_{3}\right](u, v)$. For example, another possibility is to make the following change of the parameters in $\boldsymbol{s}_{1}\left[a_{1}, c_{3}\right](u, v)$ :

$$
u \rightarrow \sqrt{\frac{5+\sqrt{5}}{8}} u+\frac{\sqrt{5}-1}{4} v \quad v \rightarrow-\frac{\sqrt{5}-1}{4} u+\sqrt{\frac{5+\sqrt{5}}{8}} v
$$

and to rotate about the third axis with an angle $\varphi$, such that

$$
\cos \varphi=\sqrt{\frac{5+\sqrt{5}}{8}} \quad \sin \varphi=\frac{-1+\sqrt{5}}{4}
$$

An analogous remark holds for the surfaces in section 4 .

Now consider the general chart $\boldsymbol{s}\left[a_{1}, a_{2}, c_{3}, d_{3}\right](u, v)$. Using (4.2) we see that it is generated via the Weierstrass formula with the functions

$$
f(z)=-\frac{25\left(c_{3}-i d_{3}\right)^{2}}{12\left(a_{1}+i a_{2}\right)} z^{3} \quad g(z)=-\frac{12\left(a_{1}+i a_{2}\right)}{5\left(c_{3}-i d_{3}\right)} z .
$$

A corresponding solution of the differential equation (2.5) is

$$
z(w)=\frac{5^{1 / 5}\left(-w^{2}\right)^{1 / 5}}{2^{2 / 5}\left(c_{3}-i d_{3}\right)^{1 / 5}}
$$

which gives the following generating function in canonical principal parameters:

$$
\tilde{g}(w)=-\frac{6.2^{3 / 5}\left(a_{1}+i a_{2}\right)}{5^{4 / 5}\left(c_{3}-i d_{3}\right)^{6 / 5}}\left(-w^{2}\right)^{1 / 5}
$$

and the following normal curvature in these parameters:

$$
\frac{1152.50^{1 / 5} \frac{a_{1}^{2}+a_{2}^{2}}{\left(c_{3}^{2}+d_{3}^{2}\right)^{6 / 5}|w|^{6 / 5}}}{\left(25+72.50^{1 / 5} \frac{\left(a_{1}^{2}+a_{2}^{2}\right)|w|^{4 / 5}}{\left(c_{3}^{2}+d_{3}^{2}\right)^{6 / 5}}\right)^{2}}
$$

Hence it follows that every two surfaces for which

$$
\frac{\left(a_{1}^{2}+a_{2}^{2}\right)^{5}}{\left(c_{3}^{2}+d_{3}^{2}\right)^{6}}
$$

is one and the same number are congruent. In particular, this implies Theorem 5.2 ,

On the other hand, using (5.5) and Theorem 2.4, we obtain

Theorem 5.4. For any shape parameters, the surface defined by $\boldsymbol{s}\left[a_{1}, a_{2}, c_{3}, d_{3}\right](u, v)$ coincides with the surface generated via the Weierstrass formula by the functions

$$
f(z)=z^{3}, \quad g(z)=z
$$


up to position in space and homothety.

Figure 5.1 represents such a surface from different points of view.
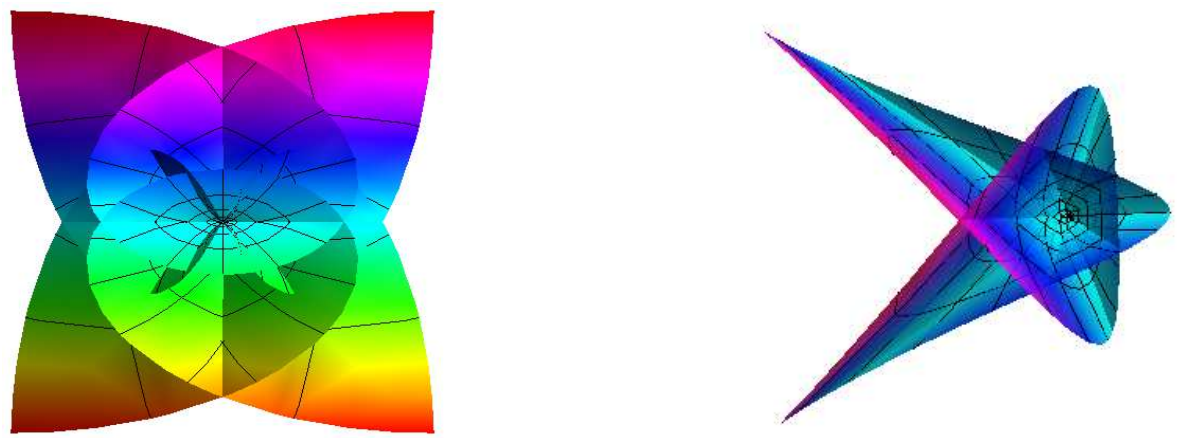

Fig. 5.1: $\boldsymbol{s}_{1}[3,0,1,0](u, v)$ for $u, v \in[-0.1,0.1]$.

At the end of this article we note that the parametric minimal surfaces of degree six in section 4 are completely different from those in section 5 . This follows from their normal curvatures and Theorem 2.1, or from the functions that generate these surfaces in canonical principal parameters and Theorem 2.3. Indeed, any of the surfaces in section 4 is generated in canonical principal parameters by a function of the form $\tilde{g}(z)=C z$, whereas any surface defined by a chart $\boldsymbol{s}\left[a_{1}, a_{2}, c_{3}, d_{3}\right](u, v)$ is generated by a function of the form $\tilde{g}(z)=C\left(-z^{2}\right)^{1 / 5}$.

\section{REFERENCES}

[1] Cosín, C., Monterde, J. Bézier surfaces of minimal area. Proc. Int. Workshop of Computer Graphics and Geom. Modelig. Lecture Notes in Comput. Sci. 2330, 2002, 72-81.

[2] Eisenhart, L.: A Treatise on the Differential Geometry of Curves and Surfaces. Ginn and company, Boston, New York, Chicago, London, 1909.

[3] Ganchev, G.: Canonical Weierstrass representation of minimal surfaces in Euclidean space. To appear. Available as arXiv:0802.2374, 2008.

[4] Gray, A., Abbena, E., Salomon, S.: Modern Differential Geometry of Curves and Surfaces with MATHEMATICA. Boca Raton, FL: CRC Press, 2006.

[5] Kassabov O.: Transition to Canonical Principal Parameters On Minimal Surfaces. Comput. Aided Geom. Design, to appear.

[6] Xu, G., Wang, G.: Parametric polynomial minimal surfaces of degree six with isothermal parameter. Lecture Notes in Comput. Sci. 4975, 2008, 329-343.

[7] Xu G., Wang G.: Quintic parametric polynomial minimal surfaces and their properties. Differential Geometry and its Applications, 28(2010), 697-704.

University of Transport, Sofia, Bulgaria

E-mail address: okassabov@abv.bg 